\title{
Free energy and entropy of a dipolar liquid by computer simulations
}

\author{
Ricardo Palomar and Gemma Sesé \\ Departament de Física, Universitat Politècnica de Catalunya, \\ Campus Nord-Mòdul B4, c/ Jordi Girona 1-3, 08034 Barcelona, Spain
}

(Dated: January 12, 2018)

\begin{abstract}
Thermodynamic properties for a system composed of dipolar molecules are computed. Free energy is evaluated by means of the thermodynamic integration technique, and it is also estimated by using a perturbation theory approach, in which every molecule is modeled as a hard sphere within a square well, with an electric dipole at its center. The hard sphere diameter, the range and depth of the well, and the dipole moment have been calculated from properties easily obtained in Molecular Dynamics simulations. Connection between entropy and dynamical properties is explored in the liquid and supercooled states by using instantaneous normal mode calculations. A model is proposed in order to analyze translation and rotation contributions to entropy separately. Both contributions decrease upon cooling, and a logarithmic correlation between excess entropy associated with translation and the corresponding imaginary frequency modes is encountered. Rosenfeld scaling law between reduced diffusion and excess entropy is tested, and the origin of its failure at low temperatures is investigated.
\end{abstract}




\section{Publishifhg INTRODUCTION}

Unraveling the role of structure and thermodynamics in the dynamics of a supercooled liquid is a long standing problem in condensed matter theory [1-4]. Free energy is a central thermodynamic property of systems in equilibrium which provides a bond between thermodynamics and statistical mechanics, gives information on the stability of a state [5], and permits the evaluation of entropy. An important connection between structure and thermodynamics is made by considering the excess entropy. Excess entropy can also be connected to transport properties, namely to the diffusivity, by means of phenomenological scaling relations $[6,7]$, which were originally motivated by the success of the variational hard-sphere thermodynamic theory. They are relevant not only-to obtain estimates for transport coefficients but also to provide guidelines for theoretical analysis.

Several approaches have been devised to compute free energies. One of the most widely used is the thermodynamic integration technique, which allows the evaluation of free energy differences $[8,9]$. Then, the free energy of a state can be obtained, provided that the free energy of a reference state is known. This methodology requires to perform a series of simulations of a system within the thermodynamic path between two states. Perturbation theories have provided useful insights in condensed matter physics for many decades [10], and they can be used to obtain free energy estimates. They allow to relate thermodynamic properties of fluids to the ones of simpler systems described by repulsive potentials, usually hard sphere potentials $[11,12]$. Short range and dipole interacions can also be included to model dipolar molecular systems[13-15].

Seminal work within the framework of the inherent structure approach [16] described glass transition as a purely configurational one, and a logarithmic relation between configurational entropy and diffusion was proposed. Excess entropy contains vibrational and configurational terms, but recent works have demonstated that vibrational entropy is fairly small for metallic glasses [17]. Excess entropy can be obtained as a multiparticle correlation expansion. Even though its behavior is well represented by the two-body approximation in some cases, it can be difficult to evaluate for molecular systems.

The main purpose of this work is to investigate correlations between thermodynamic properties and dynamics of a molecular liquid when approaching the supercooled state. The system under study is composed of diatomic molecules with a dipole moment. A pertur- 
Publishilpgive approach is used to estimate the free energy, which involves a new methodology to estimate the parameters characterizing perturbation potentials. They are evaluated so that structural and energetic properties of the system, previously obtained in Molecular Dynamics simulations, are reproduced. Entropy is also evaluated at different temperatures approaching glass transition. In order to connect entropy and short time dynamics, instantaneous normal mode (INM) analysis $[18,19]$ is undertaken. A methodology is proposed in order to obtain rotational and translational contributions to entropy separately. The validity of the pair entropy approximation, evaluated from pair radial distribution functions [20] is tested. Correlation between excess entropy and the proportion of INM imaginary frequency modes $(\mathrm{Im}-\omega)[21]$ is investigated. Rosenfeld scaling law is also checked and the origin of its failure at low temperatures is analyzed.

The paper is composed of five sections and one appendix, where some technical details concerning the perturbative approach are gathered. In section II the methods used to compute free energy and entropy are described. Details on the molecular model and calculations are included in section III. Results are discussed in section IV. Some concluding remarks are gathered in the final section.

\section{METHODS}

\section{A. Free energy computations}

For a system composed of $N$ atoms, with a hamiltonian $H\left(\mathbf{r}^{N}, \mathbf{p}^{N}\right)$

$$
H\left(\mathbf{r}^{N}, \mathbf{p}^{N}\right)=\sum_{i=1}^{N} \mathbf{p}_{i}^{2}+V\left(\mathbf{r}^{N}\right),
$$

the free energy $A$ in the canonical ensemble can be evaluated as

$$
A(N, V, T)=-k_{B} T \ln \left[\frac{1}{h^{3 N} N !} \iint \exp \left(-H\left(\mathbf{r}^{N}, \mathbf{p}^{N}\right) / k_{B} T\right) \mathrm{d}^{N} \mathbf{p} \mathrm{d}^{N} \mathbf{r}\right]
$$

The evaluation of the integral in eq. (2) is computationally challenging as it requires a complete sampling of the phase space. Then, alternative approaches have to be considered. Thermodynamic integration (TI) is one of them. By using this methodology, the free energy difference between two states can be obtained. Provided that the free energy of one of the states is known, it is possible to find out the free energy of the other. The methodology 
Publishing uires the choice of a thermodynamic path between the initial (A) and the final (B) states. Any intermediate state with potential energy $U$ is parameterized by a coupling parameter $\alpha$, so that

$$
U(\alpha)=\alpha^{k} U_{A}+(1-\alpha)^{k} U_{B}
$$

where $k$ is an integer, $U_{A}$ and $U_{B}$ are the potential energies of states $\mathrm{A}$ and $\mathrm{B}$ respectively, and $1 \leq \alpha \leq 0$. Free energy difference between states $\mathrm{A}$ and $\mathrm{B}$ can be calculated as $[8,22]$

$$
\Delta A=A_{B}-A_{A}=\int_{1}^{0}\left\langle\frac{\partial U(\alpha)}{\partial \alpha}\right\rangle d \alpha
$$

where $\langle\cdots\rangle_{\alpha}$ is the Boltzmann average. For a system of diatomic molecules, the reference state is an ideal gas within the rigid rotor approximation [23]. Then, $U_{B}=0$ and

$$
A_{B}=N k_{B} T\left[\ln \left(\frac{N}{V}\right)+\ln \left(\frac{h^{2}}{2 \pi m_{T} k_{B} T}\right)^{3 / 2}-1+\ln \left(\frac{h^{2}}{8 \pi^{2} I k_{B} T}\right)\right] .
$$

Free energy can also be estimated by means of the perturbation theory approach. According to it, a dipolar molecule can be modeled as a hard sphere particle (HS) within a square well, with an electric dipole embedded at its center. The interacting potential between two molecules with their centres of mass located at a distance $r$ can be evaluated as

$$
u(r)=u_{H S}(r)+u_{S W}(r)+u_{D D}(r)
$$

where $u_{H S}(r)$ is the HS repulsive potential

$$
u_{H S}(r)=\left\{\begin{array}{l}
\infty, r<d \\
0, \quad r>d
\end{array}\right.
$$

being $d$ the HS diameter. For $u_{S W}(r)$, the square-well potential,

$$
u_{S W}(r)= \begin{cases}-\epsilon, & d<r<\lambda d \\ 0, & r>\lambda d\end{cases}
$$

where $\lambda$ is the range of attractive forces and $\epsilon$ is the square well depth. Dipole-dipole energy can be evaluated as

$$
u_{D D}\left(\mathbf{r}, \hat{\mathbf{n}}_{1}, \hat{\mathbf{n}}_{2}\right)=-\frac{\mu^{2}}{r^{3}} D\left(\mathbf{r}, \hat{\mathbf{n}}_{1}, \hat{\mathbf{n}}_{2}\right),
$$




$$
D\left(\mathbf{r}, \hat{\mathbf{n}}_{1}, \hat{\mathbf{n}}_{2}\right)=3\left(\hat{\mathbf{n}}_{1} \cdot \hat{\mathbf{r}}\right)\left(\hat{\mathbf{n}}_{2} \cdot \hat{\mathbf{r}}\right)-\hat{\mathbf{n}}_{1} \cdot \hat{\mathbf{n}}_{2},
$$

where $\mu$ is the magnitude of the dipole embedded at the center of the molecule, $\hat{\mathbf{n}}_{i}$ is a unit vector in the direction of dipole $i$ and $\hat{\mathbf{r}}$ is a unit vector in the direction of $\mathbf{r}=\mathbf{r}_{2}-\mathbf{r}_{1}$, being $\mathbf{r}_{1}$ and $\mathbf{r}_{2}$ the positions of the centres-of-mass of molecules 1 and 2 respectively.

Within the Barker-Henderson (BH) perturbation theory framework [11, 12], the thermodynamic properties of a real system can be evaluated by means of an expansion around their values at a reference system. In the modeling of a dipolar system, an ensemble of HS can be considered as the reference system, while the square well and dipole-dipole interactions are treated as perturbations. Free energy can be evaluated as

$$
A=A_{H S}+A_{S W}+A_{D D}+A_{R}
$$

where $A_{H S}$ is the free energy of the HS system, and $A_{S W}$ and $A_{D D}$ are the contributions associated to the square well and to the dipole-dipole interactions. $A_{R}$ is included to take into account the contribution of rotational degrees of freedom

$$
\frac{A_{R}}{N k_{B} T}=\ln \left(\frac{h^{2}}{8 \pi^{2} I k_{B} T}\right)
$$

being $I$ the molecular moment of inertia. By asuming the Carnahan-Starling (CS) equation of state [24] for the HS system,

$$
\frac{A_{H S}}{N k_{B} T}=\ln \left(\frac{h^{2}}{2 \pi m k_{B} T}\right)^{3 / 2}+\ln \rho-1+\frac{4 y-3 y^{2}}{(1-y)^{2}}
$$

where $y=\pi / 6 \rho d^{3}$ is the packing fraction and $m$ is the molecular mass.

$A_{S W}$ and $A_{D D}$ can be evaluated by using Taylor power series expansions of the inverse of the temperature. For the square-well potential term,

$$
A_{S W}=\left(\frac{\epsilon}{k_{B} T}\right) A_{1, S W}+\left(\frac{\epsilon}{k_{B} T}\right)^{2} A_{2, S W}+\ldots
$$

First-order perturbation term $A_{1, S W}$ can be calculated by means of the two-body distribution function of the representative HS fluid. Second-order perturbation term $A_{2, S W}$ involves three- and four-order radial distribution functions. Nevertheless, the so called microscopic 
Publishingoroximation allows to relate this term to the two-body distribution function, as shown in the Appendix.

By neglecting third-order contributions in eq. (14), the potential energy associated to the square-well can be evaluated as

$$
E_{S W}=\left(\frac{\partial\left(\beta A_{S W}\right)}{\partial \beta}\right)=\left(\frac{\epsilon}{k_{B} T}\right) A_{1, S W}+2\left(\frac{\epsilon}{k_{B} T}\right)^{2} A_{2, S W} .
$$

For asymmetric molecules, it can be more convenient to model the molecular core as a hard prolate ellipsoid of semi-axes $a$ and $b=c$, instead of a HS. The corresponding analytical formulas for the first- and second-order perturbation terms are given in the Appendix.

By means of a perturbation theory for polar fluids $[13,14]$ it is possible to evaluate $A_{D D}$ according to

$$
A_{D D}=\left(\frac{\mu^{2}}{k_{B} T}\right) A_{1, D D}+\left(\frac{\mu^{2}}{k_{B} T}\right)^{2} A_{2, D D}+\left(\frac{\mu^{2}}{k_{B} T}\right)^{3} A_{3, D D}+\ldots
$$

where $\mu$ is the molecular electric dipole. $A_{1, D D}$ is null because of the symmetry of the interaction. The second and third terms can be evaluated analytically from structural parameters of the reference HS system (see Appendix).

Given that series (16) is slowly convergent, the sum of infinite terms can be written as a sum of Padé [14, 25]

$$
A_{D D}=\left(\frac{\mu^{2}}{k_{B} T}\right)^{2} \frac{A_{2, D D}}{1-\left(\frac{\mu^{2}}{k_{B} T}\right) \frac{A_{3, D D}}{A_{2, D D}}}
$$

The Padéapproximation is accurate at high temperatures and provides a good description of thermodynamic properties, such as free energy [25, 26].

Finally, the potential energy associated to the dipole-dipole interaction is related to the corresponding free energy perturbation terms by means of

$$
E_{D D}=3 A_{D D}-\left(\frac{\mu^{2}}{k_{B} T}\right)^{2} \frac{A_{2, D D}-2\left(\frac{\mu^{2}}{k_{B} T}\right) A_{3, D D}}{\left[1-\left(\frac{\mu^{2}}{k_{B} T}\right) \frac{A_{3, D D}}{A_{2, D D}}\right]^{2}} .
$$

It should be noted that the model requires four ad-hoc parameters, namely the HS diameter $d$, and the energetic parameters $\epsilon, \lambda$ and $\mu$. 


\section{Publishing]3. Instantaneous Normal Mode Analysis}

The potential energy of a system at a given time $t$ can be approximated as a series expansion around the configuration $\mathbf{R}_{\mathbf{o}}=\left\{\mathbf{r}_{\mathbf{o}}\right\}^{\mathbf{N}}$ at time $t_{o}$

$$
U(\mathbf{R})=U\left(\mathbf{R}_{o}\right)-\mathbf{F}\left(\mathbf{R}_{o}\right) \cdot\left(\mathbf{R}-\mathbf{R}_{o}\right)+\frac{1}{2}\left(\mathbf{R}-\mathbf{R}_{o}\right) \cdot \mathbf{D}\left(\mathbf{R}_{\mathrm{o}}\right) \cdot\left(\mathbf{R}-\mathbf{R}_{\mathbf{o}}\right)
$$

where $\mathbf{R}(t)=\{\mathbf{r}\}^{N}$ is the mass-weighted coordinates vector at time $t$. For a diatomic molecule with $5 N$ degrees of freedom, the vector force $\mathbf{F}$ is a $5 N$-dimensional vector whose components are

$$
[\mathbf{F}]_{j \mu}=-\frac{\partial U}{\partial r_{j \mu}}
$$

and $\mathbf{D}$ is the $5 N \times 5 N$ dynamical matrix

$$
[\mathbf{D}]_{j \mu, k \nu}=\frac{\partial^{2} U}{\partial r_{j \mu} \partial r_{k \nu}} .
$$

One approach to specify the mass-weighted rotation coordinates requires the use of Euler angles[18]. Then, for molecule $j$,

$$
r_{j \mu}=\left\{\begin{array}{llll}
m^{1 / 2} x_{j}, & m^{1 / 2} y_{j}, & m^{1 / 2} z_{j} & \mu=\{1,2,3\} \\
I^{1 / 2} \theta_{j}, & I^{1 / 2} \sin \theta_{o j} \phi_{j} & \mu=\{4,5\}
\end{array}\right.
$$

If $\mathbf{U}\left(\mathbf{R}_{o}\right)$ is the unitary/matrix that diagonalizes $\mathbf{D}\left(\mathbf{R}_{o}\right)$, then the eigenvalues of the dynamical matrix are given by

$$
\omega_{\alpha}^{2}=\left[\mathbf{U} \cdot \mathbf{D} \cdot \mathbf{U}^{t}\right]_{\alpha \alpha} \quad \alpha=\{1, \ldots, 5 N\}
$$

where $\mathbf{U}^{t}$ is the transpose of $\mathbf{U}$. The set of $5 N$ eigenvalues $\omega_{\alpha}^{2}$ are the squares of normal mode frequencies. For a liquid, the first derivative term $\mathbf{F}$ does not vanish, and the Hessian has negative eigenvalues, that is, Im- $\omega$ modes. Some of these modes are related to diffusion [27].

Density of states (DOS) $\rho(\omega)$ is obtained after averaging over liquid configurations

$$
\rho(\omega)=\frac{1}{5 N}\left\langle\sum_{\alpha=1}^{5 N} \delta\left(\omega-\omega_{\alpha}\right)\right\rangle .
$$


Publishing or a spectrum of frequencies normalized to unity, the fraction of real frequencies or stable modes $(k=s)$ and imaginary frequencies or unstable modes $(k=u)$ can be obtained as

$$
f_{k}=\int_{k} \rho(\omega) \mathrm{d} \omega .
$$

Translational and rotational frequencies within the DOS spectrum can be identified by considering projectors

$$
\begin{aligned}
& {\left[\mathbf{P}_{T}\right]_{j \mu, k \nu}= \begin{cases}\delta_{j k} \delta_{\mu \nu} & \mu, \nu \leq\{3\} \\
0 & \mu, \nu=\{4,5\}\end{cases} } \\
& {\left[\mathbf{P}_{R}\right]_{j \mu, k \nu}= \begin{cases}\delta_{j k} \delta_{\mu \nu} & \mu, \nu=\{4,5\} \\
0 & \mu, \nu \leq\{3\}\end{cases} }
\end{aligned}
$$

Then, $\mathbf{P}_{T}+\mathbf{P}_{R}=\mathbf{1}$. Translation and rotation spectra are given by

$$
\begin{aligned}
& \rho_{T}(\omega)=\frac{1}{5 \mathbb{N}}\left\langle\sum_{\alpha=1}^{5 N}\left[\mathbf{P}_{\mathbf{T}}^{\prime}\right]_{\alpha \alpha} \delta\left(\omega-\omega_{\alpha}\right)\right\rangle \\
& \rho_{R}(\omega)=\frac{1}{5 N}\left\langle\sum_{\alpha=1}^{5 N}\left[\mathbf{P}_{\mathbf{R}}^{\prime}\right]_{\alpha \alpha} \delta\left(\omega-\omega_{\alpha}\right)\right\rangle,
\end{aligned}
$$

where $\mathbf{P}_{T}^{\prime}$ and $\mathbf{P}_{R}^{\prime}$ result from the diagonalization of projectors whose components are defined in eqs.(26) and (27), namely

$$
\begin{aligned}
\mathbf{P}_{T}^{\prime} & =\mathbf{U} \cdot \mathbf{P}_{T} \cdot \mathbf{U}^{t} \\
\mathbf{P}_{R}^{\prime} & =\mathbf{U} \cdot \mathbf{P}_{R} \cdot \mathbf{U}^{t} .
\end{aligned}
$$

\section{Excess Entropy}

Entropy $(S)$ is related to free energy according to $S=(A-E) / T$, being $E$ the internal energy and $T$ the temperature. Excess entropy $\left(S_{e x c}\right)$ can be defined by means of

$$
S=S_{i d}+S_{e x c},
$$

where $S_{i d}$ is the entropy of an ideal gas at the same density and temperature. For a monoatomic system composed of $N$ particles of mass $m$ in a volume $V$ 


$$
\frac{S_{i d}}{N k_{B}}=-\ln \left(\frac{h^{2}}{2 \pi m k_{B} T}\right)^{3 / 2}-\ln \left(\frac{N}{V}\right)+\frac{5}{2} .
$$

$S_{\text {exc }}$ is the so called excess entropy, which can be expanded as a series [20]

$$
S_{e x c}=S_{2}+S_{3}+\ldots
$$

$S_{n}$ can be obtained from integration of n-particle distribution functions. This approach has been used to estimate the entropy of simple liquids [20, 28, 29]. Although the convergence of the series expansion (32) is slow, specially at high densities, sometimes it is possible to approximate $S_{\text {exc }} \approx S_{2}$ because the three body contribution $S_{3}$ partially cancels four-body and higher order terms. Indeed, several authors found that $S_{2}$ accounts for around $85 \%$ of the total excess entropy [20, 29]. For a homogeneus and isotropic liquid, the two-body contribution $S_{2}$ can be evaluated as

$$
\frac{S_{2}}{N k_{B}}=-\frac{1}{2} \rho \int[g(r) \ln g(r)-g(r)+1] \mathrm{d} \mathbf{r},
$$

where $\rho$ is the number density and $g(r)$ is the pair radial distribution function.

A new approach is proposed in order to obtain rotational and translational contributions to the total entropy of a molecular system separately. It assumes that the system is composed by a gas-like subsystem composed of $N_{g}=q N$ molecules and a solid-like subsystem containing $N_{s}=(1-q) N$ molecules, being $N$ the total number of molecules in the sample. Then, the total entropy is the superposition of gas-like $\left(S_{g}(q)\right)$ and solid-like $\left(S_{s}(q)\right)$ contributions

$$
S=S_{g}(q)+S_{s}(q)
$$

$S_{g}(q)$ includes low frequency processes which are mainly associated with diffusion. For a system of diatomic molecules, it is evaluated by using the free rigid rotor approximation

$$
\frac{S_{g}(q)}{N k_{B}}=q\left[-\ln \left(\frac{h^{2}}{2 \pi m k_{B} T}\right)^{3 / 2}-\ln \left(\frac{q N}{V}\right)-\ln \left(\frac{h^{2}}{8 \pi^{2} I k_{B} T}\right)+\frac{7}{2}\right] .
$$

$S_{s}(q)$ is evaluated from the real frequencies branch of spectrum $\rho(\omega)$,

$$
\frac{S_{s}(q)}{N k_{B}}=5(1-q) \int_{\text {real }}\left[\frac{x_{o}}{\exp \left(x_{o}\right)-1}-\ln \left(1-\exp \left(-x_{o}\right)\right)\right] \rho(\omega) \mathrm{d} \omega
$$


Publishiwgere $\rho(\omega)$ has been normalized to unity, and $x_{o}=\hbar \omega / k_{B} T$. For $q=1$, the entropy of the system equals that of a gas, while for $q=0$ it is that of a crystal. From eq. (34), and using results from eqs. (35) and (36), it is possible to obtain the gas-weight parameter $q$ at each temperature.

This approach is inspired by the 2PT methodology [30], although some differences are to be noted. First, they obtain spectra after Fourier transforming velocity autocorrelation functions, whereas DOS spectra evaluated from INM analysis are used in our approach. In addition, their HS reference system is replaced by the rigid rotor model because the latter allows to consider one single weight parameter $q$ for both translation and rotation terms. Finally, the $q$ parameter is defined on purely thermodynamic grounds, whereas a dynamical definition is used in the 2PT approach.

Total entropy includes a translation $\left(S^{T}\right)$ and a rotation $\left(S^{R}\right)$ contribution $\left(S=S^{T}+S^{R}\right)$. They can be obtained by means of

$$
S^{\alpha}=S_{g}^{\alpha}(q)+S_{s}^{\alpha}(q)
$$

where $\alpha=\{T, R\}$. For the gas-like subsystem,

$$
\begin{gathered}
S_{g}^{T}(q)=q N k_{B}\left[-\ln \left(\frac{h^{2}}{2 \pi m k_{B} T}\right)^{3 / 2}-\ln \left(\frac{q N}{V}\right)+\frac{5}{2}\right] \\
S_{g}^{R}(q)=q N k_{B}\left[-\ln \left(\frac{h^{2}}{8 \pi^{2} I k_{B} T}\right)+1\right] .
\end{gathered}
$$

Entropy associated with the solid-like subsystem is evaluated by means of

$$
S_{s}^{\alpha}(q)=n_{\alpha}(1-q) N k_{B} \int_{\text {real }}\left[\frac{x_{o}}{\exp \left(x_{o}\right)-1}-\ln \left(1-\exp \left(-x_{o}\right)\right)\right] \rho_{\alpha}(\omega) \mathrm{d} \omega,
$$

where $n_{T}=3, n_{R}=2$ and $x_{o}=\hbar \omega / k_{B} T$, and only real frequencies branches of $\rho_{\alpha}(\omega)$ are used.

\section{MOLECULAR MODEL AND DETAILS OF THE CALCULATIONS}

The system under investigation $(\mathrm{MeO})$ is composed of rigid neutral molecules, with two interacting sites whose masses are those of oxygen $(\mathrm{O})$ and of the methyl (Me) group. Charges have been assigned to both sites so that the molecular dipole equals that of methanol 
Publishinglecules $(2.22 \mathrm{D})$. Then, the molecular model is very similar to the one used to simulate methanol with the exception of the ability to set up hydrogen bonds.

Short range interactions have been evaluated by means of the OPLS potential [31]. Then, the potential energy for each pair of sites belonging to different molecules consists of a Lennard-Jones part and a coulombic term

$$
u\left(r_{i j}\right)=4 \epsilon_{i j}\left[\left(\frac{\sigma_{i j}}{r_{i j}}\right)^{12}-\left(\frac{\sigma_{i j}}{r_{i j}}\right)^{6}\right]+\frac{q_{i} q_{j}}{r_{i j}} .
$$

Potential parameters are gathered in table I. The model was proposed by Hirata and Levy[32], and it has been investigated at liquid [33] and supercooled conditions [34-36]. Bond lenghts have been kept at their equilibrium values by means of the Shake method [8]. Ewald summation [8] has been used in the calculation of electrostatic interactions.

The system consists of $N=1000$ molecules and it has been equilibrated in the canonical ensemble at $T=298 \mathrm{~K}$ with a density of $0.787 \mathrm{~g} / \mathrm{cm}^{3}$. Then, the sample has been quenched at constant pressure in a stepwise manner with $\Delta T=-5 \mathrm{~K}$. Every change in temperature has been followed by a thermalization period in the $(N, P, T)$ ensemble. Along the cooling process, some temperatures have been selected. For each of them, additional equilibration times have been performed, ranging from 250 ps to 1750 ps at the highest and the lowest temperature respectively. Subsequently, production runs have been carried out in the $(N, V, T)$ ensemble, which have been used to evaluate radial distribution functions, diffusion coefficients and energetic values. Temperature and pressure have been controlled by means of the algorithm proposed by Berendsen et al. [37].

Free energy has been evaluated with the TI technique and also by using a perturbative approach. For a model system, convergence of both methodologies is particularly relevant. As for the TI technique, evaluation of the integrand in eq.(4) requires to perform a series of MD simulations, where the interacting potentials between sites $i$ and $j$ depend on the $\alpha$ parameter as follows [38]

$$
\begin{gathered}
u\left(\alpha, r_{i j}\right)=4 \epsilon_{i j}\left[\frac{1}{\left[\alpha_{o}(1-\alpha)^{m}+\left(r_{i j} / \sigma_{i j}\right)^{6}\right]^{2}}-\frac{1}{\alpha_{o}(1-\alpha)^{m}+\left(r_{i j} / \sigma_{i j}\right)^{6}}\right]+ \\
+\frac{q_{i} q_{j}}{\left[\alpha_{o}(1-\alpha)^{m}+r_{i j}^{2}\right]^{1 / 2}} \\
U_{A}=\sum_{i<j} u\left(\alpha, r_{i j}\right) .
\end{gathered}
$$


Publishiwg have considered $m=2$ and $\alpha_{o}=0.5$. In addition, a non-linear scaling with $k=4$ has been used in eq.(3). Previous works have proven that these values ensure avoidance of singularities and reasonable computational times for a variety of liquids [39].

MD simulations with different $\alpha$ values have been performed ( $\Delta \alpha=0.1$ when approaching the ideal state and $\Delta \alpha=0.05$ otherwise) in order to obtain derivatives of the potential energy. Twenty simulations starting from independent equilibrated configurations have been undertaken for each $\alpha$-point, and each of them has been followed by a 30 ps production run. A cubic spline fitting has been used to perform numerical integration in eq.(4). No differences have been obtained when considering a 6-degree polynomial fitting procedure.

In order to undertake INM analysis, smaller samples of $N=216$ molecules have been simulated. For each temperature, we have selected 100 equally spaced independent configurations, from which the hessian has been calculated and diagonalized to obtain eigenfrequencies. Time intervals between configurations range from 1 ps at room temperature to 20 ps at the lowest analyzed temperature. No significant dependence on molecular coordinates has been observed for $\mathrm{MeO}$, in agreement with previous findings on linear rigid molecules [40].

\section{RESULTS}

\section{A. Free Energy}

Results for the derivative of the potential energy, integrand of eq.(4) are displayed in figure 1. It is apparent that changes are more abrupt for larger $\alpha$ values, and that no discontinuity is observed. Free energy difference $\Delta A$ obtained by the TI technique is -1.91 $\mathrm{kcal} / \mathrm{mol}$, and it/is obtained from eq. (5) that $A_{B}=-9.24 \mathrm{kcal} / \mathrm{mol}$, which lead to $A=$ $-11.15 \mathrm{kcal} / \mathrm{mol}$ for $\mathrm{MeO}$.

In order to analyze perturbation theory predictions, it is necessary to estimate the diameter for the HS model associated with the MeO molecule, which will be evaluated taking

into account the effective hard core diameters for $\mathrm{O}\left(d_{O}\right)$ and $\mathrm{Me}\left(d_{M e}\right)$ sites. They can be obtained from the soft-core diameters $\sigma_{O}$ and $\sigma_{M e}[11,12]$

$$
d_{i}=\int_{0}^{\sigma_{i}}\left[1-\exp \left(-\frac{u_{L J}^{(i)}(r)}{k_{B} T}\right)\right] \mathrm{d} r, \quad i=\{\mathrm{O}, \mathrm{Me}\}
$$


Publishi冈gere $u_{L J}^{(i)}(r)$ is the Lennard-Jones potential associated with a fluid of $i$-sites. By using the values for the parameters $\left(\varepsilon_{i}, \sigma_{i}\right)$ reported in table $\mathrm{I}$, it is obtained that $d_{O}=2.89 \AA$ and $d_{M e}=3.57 \AA$.

Diatomic molecules can be modeled as fused spheres with their centers located at a distance equal to the bond length $(1.43 \AA)$. The molecular HS effective diameter is evaluated by considering that its volume should equal that of the fused spheres $\left(V=29.96 \AA^{3}\right)$. It is obtained that $d=3.85 \AA$ which leads to a packing fraction of $y=0.443$. When $\mathrm{MeO}$ molecules are modeled as hard ellipsoids ( $\mathrm{HE}$ ), the length of their major axis is $2 a=d_{O} / 2+$ $l+d_{M e} / 2=4.63 \AA$, and that of the minor axis is evaluated so that the packing fraction is kept constant, leading to $2 b=3.52 \AA$.

We have evaluated the range $\lambda$ for the square-well potential (eq. (8)) by fixing that the coordination number for the HS system should equal that obtained in MD for the diatomic fluid. Then,

$$
4 \pi \rho \int_{0}^{\lambda d} r^{2} g_{C O M}(r) \mathrm{d} r=4 \pi \rho \int_{d}^{\lambda d} r^{2} g_{H S}(r) \mathrm{d} r
$$

where $g_{C O M}(r)$ is the molecular center of mass $(\mathrm{COM})$ radial distribution function and $g_{H S}(r)$ is the HS system radial distribution function evaluated as shown in the Appendix. It is apparent in figure 2 that $g_{H S}(r)$ is a good approximation for $g_{C O M}(r)$ at distances beyond the first maximum. It has been obtained that $\lambda=1.51$. In addition, the square-well potential depth, $\epsilon$, has been evaluated by means of eq.(15), by fixing that $E_{S W}=E_{L J}$. It has been obtained in MD simulations that the energy associated to LJ interactions for the MeO system is $E_{L J}=-2.55 \mathrm{kcal} / \mathrm{mol}$. For the HS model, it turns out that $\left(\epsilon / k_{B}\right)_{H S}=211.5$. When considering the HE model, it is obtained that $\left(\epsilon / k_{B}\right)_{H E}=203.4$.

Perturbative coefficients $A_{1, S W}, A_{2, S W}$ are gathered in table II, when considering either the HS model (eqs. (A1)-(A6)) or the HE one (eqs. (A12)-(A13)). In spite of small differences between partial terms, both HS and HE models lead to the same square-well potential contribution to the free energy.

With regard to the dipole-dipole perturbation terms, it results from eq. (A16) that $A_{2, D D} /\left(k_{B} T\right)^{2}=-0.109 \mathrm{kcal} \mathrm{mol}{ }^{-1} \mathrm{D}^{-4}$, and, from eq.(A17), that $A_{3, D D} /\left(k_{B} T\right)^{3}=0.030$ $\mathrm{kcal} \mathrm{mol} \mathrm{m}^{-1} \mathrm{D}^{-6}$. The electrostatic contribution to the potential energy of $\mathrm{MeO}$ amounts $E_{C}=-1.38 \mathrm{kcal} / \mathrm{mol}$ according to $\mathrm{MD}$ simulation. By fixing that $E_{D D}=E_{C}$ in eq. (18), it 
Publishilisgossible to evaluate the dipolar moment embedded in the HS model. We have obtained that $\mu=2.10 \mathrm{D}$, which is about $5 \%$ lower than that of $\mathrm{MeO}$ molecules in MD.

As shown in table II, differences of about $1 \%$ have been obtained for the free energy when modeling molecules as HE instead of HS. It is apparent that the value provided by perturbation theory is about $-10.46 \mathrm{kcal} / \mathrm{mol}$. It deviates from the exact value obtained by thermodynamic integration only about $6 \%$.

\section{B. Excess Entropy}

Entropy has been evaluated by using the thermodynamic relation

$$
S(T)=S\left(T_{o}\right)+\int_{T_{0}}^{T} \frac{C_{p}\left(T^{\prime}\right)}{T^{\prime}} \mathrm{dT}^{\prime} .
$$

$C_{p}(T)$ have been obtained from numerical temperature derivatives of enthalpy, which led to $C_{p}=0.04 T^{-0.2}$ [41]. The value for $S\left(T_{0}\right)$ is $29.19 \mathrm{cal} \mathrm{mol}^{-1} \mathrm{~K}^{-1}$, being the reference temperature $T_{o}=298 \mathrm{~K}$. It has been evaluated by using the TI free energy value and the computed total energy at $T_{o}$, as obtained in MD simulations $(E=-2.45 \mathrm{kcal} / \mathrm{mol})$, by following $S=(E-A) / T$. Entropy values are summarized in table III.

Results for the total DOS spectrum, $\rho(\omega)$, at the two limiting temperatures are displayed in figure 3. Translational $\rho_{T}(\omega)$ and rotational $\rho_{R}(\omega)$ contributions are also shown. At room temperature, the real frequencies lobe is larger than the one associated with imaginary frequencies for the translational spectrum, whereas the opposite applies to the rotational one. The fraction of imaginary frequencies is related to the probability of finding the system on a potential energy surface region with a negative curvature, leading to a larger diffusion. The relative large size of the unstable modes contribution to the rotational spectrum is consistent with access to larger regions in the rotational phase space. Upon cooling, the total spectrum narrows. Nevertheless, the rotational contribution is only weakly temperature dependent. This is consistent with previous results showing that rotation is less hindered than translation at the analyzed temperatures [42], even though this contribution probably includes non-diffusive modes.

By using entropy values, equation (34) has been solved in order to evaluate the gas-like parameter $q$ at each temperature. As shown in table III, $q$ decreases upon cooling so that entropy approaches that of a solid. By considering the same $q$ value for both translation 
Publishing $g^{R}$ ) and rotation $\left(S^{R}\right)$ contributions, it is possible to evaluate them. $S^{T}$ and $S^{R}$ are also listed in table III, being $S^{T}$ larger at all temperatures. By using the analogs to eqs.(38) to (40) for the energy [23], it is also possible to obtain translational and rotational contributions to the energy of the system. Differences with simulation results are lower than $2 \%$ at all temperatures, which indicates that using the same $q$ parameter for both translation and rotation contributions is a good approximation.

Excess entropy associated with translation $S_{e x c}$ is defined as the difference between translational entropy and the ideal gas term at the same density-and temperature

$$
S_{e x c}=S^{T}-S_{i d}^{T}
$$

where the index $T$ has been neglected for the sake of simplicity. The two-phase contribution to entropy underlying eq. (37) allows to connect $q$ and $S_{\text {exc }}$. Taking into account that $S_{i d}^{T}=S_{g}^{T}(q=1)$,

$$
S_{\text {exc }}=S_{g}^{T}(q)+S_{s}^{T}(q)-S_{g}^{T}(1) .
$$

We have checked whether it is possible to approximate $S_{\text {exc }}$ by the two-body excess entropy $S_{2}$, defined by equation (33) and evaluated by using the COM radial distribution function. It is obtained that 3-body and higher contributions account for $15 \%$ of $s_{e x c}^{*}$. In addition, it is apparent in figure 4 that a linear dependence exists between $s_{e x c}^{*}=S_{e x c} / N k_{B}$ and $s_{2}^{*}=S_{2} / N k_{B}$ at all temperatures. Then, the behavior of both quantities is qualitatively the same, as obtained for different model systems [43, 44], and the trends in $s_{e x c}^{*}$ with $T$ are well represented by $s_{2}^{*}$.

We have tested the empirical scaling law proposed by Rosenfeld [7] which relates the dimensionless or reduced translational diffusivity $D^{*}$ to the reduced excess entropy according to

$$
D^{*}=D \frac{\rho^{1 / 3}}{\left(k_{B} T / m\right)^{1 / 2}}=a \exp \left(b s_{e x c}^{*}\right),
$$

where $D$ is the diffusion coefficient, and $a$ and $b$ depend on the potential. As shown in figure 5 , the relation is fulfilled for $T>178 \mathrm{~K}$, with $a=0.65$ and $b=1.08$. It has been reported that the origin of the Rosenfeld law breakdown in the supercooled region can result from the appearance of dynamic heterogeneities and cooperative dynamics $[1,45,46]$. 
Publishihgleed, this behavior was previously demostrated for the system under investigation $[47,48]$.

Nevertheless, it would also be possible that the isobaric cooling process could lead to density dependent $a$ and $b$ parameters $[1,49]$.

The validity of the Rosenfeld law has been linked to an Arrhenius behavior for the diffusion coefficient of a strong liquid, resulting in a linear dependence between $s_{\text {exc }}^{*}$ and $1 / T$ [50]. This linearity is apparent in figure 6 for $298 \mathrm{~K}<T<158 \mathrm{~K}$, the temperature range at which both the Arrhenius and the Rosenfeld law are valid. Then, this connection also applies for fragile liquids.

The Mori-Zwanzig [51] formalism allows to find a relationship between $D$ and the memory function $K(t)$

$$
D=\frac{k_{B} T / m}{\tilde{K}(0)}=\frac{k_{B} T / m}{\int_{0}^{\infty} K(t) d t}
$$

The memory function $K(t)$ can be split into two contributions $[52,53]$

$$
K(t)=K_{B}(t)+K_{M C}(t)
$$

where $K_{B}(t)$ is the binary term, which can be related to the uncorrelated collisions between neighbouring molecules, and deseribes the short time behavior of $K(t) . K_{M C}(t)$ is the so called mode coupling term. It takes into account contributions associated with multiple collisions, and describes $K(t)$ properties on intermediate time scales. Accordingly, $D$ can be split into two contributions $D_{B}$ and $D_{C}$, associated with $K_{B}(t)$ and $K_{M C}(t)$, respectively, so that

$$
\frac{1}{D}=\frac{1}{D_{B}}+\frac{1}{D_{C}}
$$

$D_{B}$ can be evaluated by considering a gaussian memory function for the binary term [35], and, by using $D$ and $D_{B}$, it is possible to obtain $D_{C}$. These quantities are gathered in table IV, and it is apparent that $D$ approaches $D_{B}$ at high temperatures, whereas it is very close to $D_{C}$ at low temperatures. It is interesting to check whether the range of applicability of the Rosenfeld scaling law is the same for both contributions.

A Rosenfeld scaling law for $D_{B}^{*}$ can be written

$$
D_{B}^{*}=D_{B} \frac{\rho^{1 / 3}}{\left(k_{B} T / m\right)^{1 / 2}}=a_{1} \exp \left(b_{1} s_{B}^{*}\right),
$$


Publishiwgere $s_{B}^{*}$ is the excess entropy associated with binary, short range interactions. It has been taken equal to that of a HS system with a CS equation of state [24]

$$
s_{B}^{*}=\frac{S_{B}}{N k_{B}} \approx-\frac{4 y_{B}-3 y_{B}^{2}}{\left(1-y_{B}\right)^{2}},
$$

where $y_{B}=\pi \rho d_{B}^{3} / 6$ is the packing fraction. The HS effective diameter $d_{B}$ has been evaluated by considering the Ruckenstein-Liu [54] equation for the diffusivity at moderate and high densities

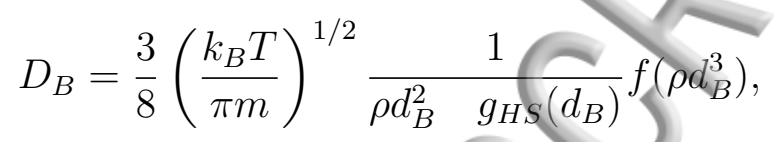

where

$$
f\left(\rho d_{B}^{3}\right)=1+0.94605\left(\rho d_{B}^{3}\right)^{1.5}+1.4022\left(\rho d_{B}^{3}\right)^{3}-5.6898\left(\rho d_{B}^{3}\right)^{5}+2.6626\left(\rho d_{B}^{3}\right)^{7}
$$

and $g_{H S}\left(d_{B}\right)$ is the HS radial distribution function at the contact distance that results from the CS equation [24], $g_{H S}\left(d_{B}\right)=\left(1-0.5 y_{B}\right) /\left(1-y_{B}\right)^{3}$. Figure 6 also displays $s_{B}^{*}$ against $1000 / T$.

It should be noted that eq.(53) is an approximation to the excess entropy associated with binary interactions because the latter are instantaneous in HS systems, whereas they occur on a time range $\tau_{B} \approx 0.06 \mathrm{ps}$ if a gaussian memory function is considered [35]. Nevertheless, this time scale is much smaller than the one associated with mode-coupling processes.

$D_{B}^{*}$ is displayed against $s_{B}^{*}$ in figure 7. It is apparent that the Rosenfeld scaling law for binary/interactions (eq.(52)) is fulfilled at all temperatures. It has been obtained that $a_{1}=0.88$ and $b_{1} \neq 0.75$, which proves that they do not depend on density. On the contrary, a Rosenfeld scaling law is not fulfilled by the mode-coupling diffusivity coefficient. Alternatively, $D_{C}^{*}$ is displayed against $s_{e x c}^{*} / s_{B}^{*}$ in figure 8 and a linear behavior is apparent. It is found that

$$
D_{C}^{*}=D_{C} \frac{\rho^{1 / 3}}{\left(k_{B} T / m\right)^{1 / 2}}=a_{2} \exp \left[b_{2}\left(s_{e x c}^{*} / s_{B}^{*}\right)\right],
$$

where $a_{2}=999$ and $b_{2}=-12.3$, for the temperature range under study. It is interesting to note that the ratio $s_{e x c}^{*} / s_{B}^{*}$ is an indicator for the Rosenfeld breakdown of the diffusivity. 
This manuscript was accepted by J. Chem. Phys. Click here to see the version of record.

Publishihgleed, taking into consideration results shown in figure 6, it is remarkable that the temperature at which $s_{e x c}^{*} \approx s_{B}^{*}$ is the crossover temperature associated with the Rosenfeld regime. That is, the scaling law is not fulfilled when $s_{e x c}^{*} / s_{B}^{*}>1$, which coincides with the mode coupling term being greater than the binary term (see eq.(51)). These findings show that the breakdown of the Rosenfeld scaling law for the diffusivity $D^{*}$ in the deep supercooled state results from the behavior of the mode-coupling contribution to the diffusion coefficient.

Taking into account eq. (47) and that the harmonic term $S_{s}^{T}$ is connected with the fraction of stable modes $f_{s}=1-f_{u}$, it seems reasonable that $s_{e x c}^{*}$ and $f_{u}$ are related. Both quantities are displayed in figure 9 and a correlation between them is apparent. Specifically, a linear dependence is obtained between $s_{e x c}^{*}$ and $\ln \left(f_{u}\right)$ for all analyzed temperatures (see inset of figure 9). Then, when analyzing translation, the number of available states to the system is strongly related to the proportion of unstable modes. An analogous relationship was encountered when considering configurational entropy and overall proportion of imaginary frequency modes[55]. Then, when the Rosenfeld law (48) is fulfilled, a linear relationship occurs between $\ln D^{*}$ and $\ln f_{u}$. Even though not all unstable modes are related to diffusive processes $[27,56-58]$, this result implies that the fraction of diffusive modes should be proportional to the fraction of unstable modes, at the temperatures under study.

\section{CONCLUDING REMARKS}

A perturbative approach has been used to obtain good estimates of free energy for molecular dipolar systems. Every dipolar molecule is modeled as a hard sphere within a square well with a dipole moment embedded at its center. The hard sphere diameter can be evaluated by fixing the packing fraction. Parameters that characterize the square well can be obtained by fixing the coordination number and the energy associated to short range interactions. The electric dipole can be estimated by fixing the electrostatic energy. All these parameters can be obtained using results of a single molecular dynamics simulation, whereas thermodynamic integration technique requires to perform a series of simulations.

A methodology intended to evaluate translational and rotational contributions to excess entropy has been proposed. It requires both translational and rotational spectra, which have been obtained by instantaneous normal modes analysis. It is remarkable that the rotational density of states is only weakly temperature dependent. As for the translational spectrum, 
Publishithe imaginary lobe is strongly diminished and the real part slightly shifts towards larger frequencies upon cooling, which is consistent with a preferential intrabasin energy landscape dynamics in the supercooled state. The approach states that both contributions to the entropy contain gas-like and solid-like contributions. It has been found that excess entropy decreases upon cooling, and that its translational part and the fraction of imaginary frequency modes are correlated. The two-body approximation for the excess entropy accounts for about $85 \%$ of its translational contribution, even in the supercooled state.

The Rosenfeld scaling law between excess entropy and-diffusion has been tested, and its validity within the same temperature range as that of the Arrhenius law for diffusion has been confirmed. It has also been demonstrated that the breakdown of the law in the supercooled state stems from mode-coupling terms.

\section{ACKNOWLEDGMENTS}

Finantial support of MINECO (Project FIS2015-66879-C2-1-P) is acknowledged. 


\section{PublishingAppendix A: Square well and dipole-dipole perturbation terms}

First-order perturbation term for the square well potential, can be related to the two-body distribution function of the representative HS fluid [11] by means of

$$
\frac{A_{1, S W}}{N k_{B} T}=-12 y L(\lambda, y)
$$

where

$$
L(\lambda)=\int_{1}^{\lambda} g_{H S}(x) d x
$$

being $x=r / d$ the reduced distance, and $g_{H S}(r)$ the radial distribution function of the HS system. The later can be evaluated by means of the semi-empirical equation [59]

$$
g_{H S}(x)= \begin{cases}1+x^{-1} \sum_{m=1}^{3} \frac{y m}{(1-y)^{m}} g_{m}(x), & 1 \leq x<3 \\ 1, & x \geq 3 .\end{cases}
$$

where $g_{m}(x)$ are polynomials of non linear base functions, which result in a rapidly convergent expansion

$$
g_{m}(x)=\sum_{n=0}^{3} C_{m n}\left(s_{1}-s_{1}^{-4}\right)^{n}, \quad 1 \leq x<2
$$

with $s_{1}=\exp (x-1)$ and

$$
g_{m}(x)=\sum_{n=0}^{3} D_{m n}\left(s_{2}-s_{2}^{-7}\right)^{n}, \quad 2 \leq x<3
$$

where $s_{2}=\exp (x-2) . C_{m n}$ and $D_{m n}$ are chosen so that theoretical values of $g_{H S}(x=1)$ and $g_{H S}(x=2)$ given by the CS equation of state [24] are reproduced. Then, $L(\lambda)$ is basically a function of the packing fraction $y$.

Second-order perturbation term $A_{2, S W}$ involves three- and four-order radial distribution functions. The microscopic approximation by Barker and Henderson [11] leads to

$$
\frac{A_{2, S W}}{N k_{B} T}=-6 Q_{H S} y \frac{\partial}{\partial y}[y L(\lambda, y)]
$$

$Q_{H S}$ is the compressibility of the HS system, which can be evaluated from the CS equation of state

$$
\frac{P_{H S}}{\rho k_{B} T}=1+\frac{4 y-2 y^{2}}{(1-y)^{3}}=\frac{1+y+y^{2}-y^{3}}{(1-y)^{3}},
$$


Publishiwgere $P_{H S}$ is the pressure of the HS system. Then,

$$
Q_{H S}=k_{B} T\left(\frac{\partial \rho}{\partial P}\right)_{H S}=\frac{(1-y)^{4}}{1+4 y+4 y^{2}-4 y^{3}+y^{4}}=\left[1+\frac{8 y-2 y^{2}}{(1-y)^{4}}\right]^{-1} .
$$

If molecules are modeled as hard prolate ellipsoids, eqs. (A1)-(A6) should be slightly modified [60]. Being $a$ and $b=c$ the hard ellipsoid (HE) semi-axes, and $\chi_{o}=a / b$ and $\chi=\left(\chi_{o}^{2}-1\right) /\left(\chi_{o}^{2}+1\right)$, the equation of state for the HE system is

$$
\frac{P_{H E}}{\rho k_{B} T}=1+\frac{4 y-2 y^{2}}{(1-y)^{3}} F_{1}(\chi)
$$

where $P_{H E}$ is the pressure, $y=4 / 3 \pi \rho a b^{2}$ is the packing fraction and

$$
F_{1}(\chi)=\left(1-\chi^{2}\right)^{-1 / 2}\left[1-\frac{1}{6} \chi^{2}-\frac{1}{40} \chi^{4}-\frac{1}{112} \chi^{6}+\ldots\right] .
$$

By integrating $P_{H E}$ over volume, free energy of the HE fluid can be evaluated as

$$
\frac{A_{H E}}{N k_{B} T}=\ln \left(\frac{h^{2}}{2 \pi m k_{B} T}\right)^{3 / 2}+\ln \rho-1+\frac{4 y-3 y^{2}}{(1-y)^{2}} F_{1}(\chi) .
$$

Perturbation terms can be calculated by following

$$
\begin{aligned}
& \frac{A_{1, S W}}{N k_{B} T}=-12 y\left(1-\chi^{2}\right)^{-1 / 2} L(\lambda, y) \\
& \frac{A_{2, S W}}{N k_{B} T}=-6 Q_{H E} y \Delta(\chi) \frac{\partial}{\partial y}[y L(\lambda, y)]
\end{aligned}
$$

with

$$
\begin{gathered}
Q_{H E}=k_{B} T\left(\frac{\partial \rho}{\partial P}\right)_{H E}=\left[1+\frac{8 y-2 y^{2}}{(1-y)^{4}} F_{1}(\chi)\right]^{-1} \\
\Delta(\chi)=\left(1-\chi^{2}\right)^{-1 / 2}\left[1+\frac{1}{6} \chi^{2}+\frac{3}{40} \chi^{4}+\frac{5}{112} \chi^{6}+\ldots\right] .
\end{gathered}
$$

As for the series expansion of the dipole-dipole potential, its relevant terms are given by[14]

$$
\frac{A_{2, D D}}{N k_{B} T}=-\frac{\rho}{4} \int \frac{D^{2}(12)}{r^{6}} g_{H S}(r) d \mathbf{r}=-\frac{\rho}{6} \int \frac{g_{H S}(r)}{r^{6}} \mathrm{~d} \mathbf{r}
$$




$$
\frac{A_{3, D D}}{N k_{B} T}=\frac{\rho^{2}}{6} \int \frac{D(12) D(13) D(23)}{r_{12} r_{13} r_{23}} g_{H S}(123) d \mathbf{r}_{2} d \mathbf{r}_{3}=\frac{\rho^{2}}{6} I_{D D D},
$$

where $g_{H S}(123)$ is the triplet distribution function of the HS fluid, and

$$
I_{D D D}=\int \frac{1+3 \cos \theta_{1} \cos \theta_{2} \cos \theta_{3}}{\left(r_{12} r_{13} r_{23}\right)^{3}} g_{H S}(123) d \mathbf{r}_{2} d \mathbf{r}_{3} \text {. }
$$

$\left(\theta_{1}, \theta_{2}, \theta_{3}\right)$ are the three angles of the triangle formed by $\left(\mathbf{r}_{\mathbf{1 2}}, \mathbf{r}_{\mathbf{1 3}}, \mathbf{r}_{\mathbf{2 3}}\right)$. For dipolar HS, it was found that eq.(A18) depends only on the packed fraction $y$ and that it can be evaluated by $[61]$

$$
I_{D D D}=\frac{5 \pi^{2} d^{6}}{3} \frac{1+2.15344 y+2.04964 y^{2}}{1-0.10495 y+0.48629 y^{2}}
$$

[1] M. Agarwal, M. Singh, R. Sharma, M. Parvez Alam and C. Chakravarty, J. Phys. Chem. B 114, 6995 (2010).

[2] A. Barros de Oliveira, E. Salcedo, C. Chakravarty and M. C. Barbosa, J. Chem. Phys. 132, $234509(2010)$.

[3] B.Bagchi, Molecular Relaxation in Liquids (Oxford, New York, 2012).

[4] F. W. Starr, J. F. Douglas and S. Sastry, J. Chem. Phys. 138, 12A541 (2013).

[5] Ch. Chipot and A. Pohorille, eds., Free Energy Calculations: Theory and Applications in Chemistry and Biology (Springer Verlag, Berlin, 2007).

[6] M. Dzugutov, Nature 381, 137 (1996); I. Yokohama, Physica B 254, 172 (1998).

[7] Y. Rosenfeld, Phys. Rev. A 15, 2545 (1977); Y. Rosenfeld, J. Phys.: Condens. Matter 11, 5415 (1999)

[8] D. Frenkel and B. Smit, Understanding Molecular Simulation (Academic Press, London, 2002).

[9] W.F, van Gunsteren, X. Daura and A.E. Mark, Helvetia Chimica Acta, 85, 3133 (2002).

[10] J.P. Hansen and I.R. McDonald, Theory of simple liquids (Academic Press, London, 2013).

[11] J.A. Barker and D. Henderson, J. Chem. Phys. 47, 2856 (1967).

[12] J.A. Barker and D. Henderson, Rev. of Mod. Physics 48, 587 (1976).

[13] G. Stell, J.C. Rasaiah, and H. Narang, Mol. Phys. 23, 393 (1972).

[14] G. Stell, J.C. Rasaiah, and H. Narang, Mol. Phys. 27, 1393 (1974). 
D. Henderson, Cond. Matter Phys. 14, 33001 (2011).

[16] J. H. Gibbs and E. A. DiMarzio, J. Chem. Phys. 28, 373 (1958); G. Adam and J. H. Gibbs, J. Chem. Phys. 43, 139 (1965).

[17] H.L.Smith, C.W. Li, A.Hoff, G.R. Garrett, D.S. Kim, F.C. Yang, M.S. Lucas, T. Swan-Wood, J.Y.Y. Lin, M.B. Stone, D.L. Abernathy, M.D. Demetriou and B.Fultz, Nature Physics 13, 900 (2017).

[18] M. Buchner, B. M. Ladanyi and R. M. Stratt, J. Chem. Phýs. 97, 8522 (1992).

[19] T. Keyes, G.V. Vijayadamodar and U. Zurcher, J. Chem. Phys. 106, 4651 (1997).

[20] A. Baranyai and D.J. Evans, Phys. Rev. A 40, 3817(1989).

[21] S.H. Chakraborty and Ch. Chakravarty, J. Chem. Phys. 124, 014507 (2006).

[22] J.G. Kirkwood, J. Chem. Phys. 3, 300 (1935).

[23] D. A. McQuarrie, Statistical Mechanics (University Science Books, California, 2000).

[24] N.F. Carnahan and K.E. Starling, J. Chem. Phys. 51, 635 (1969).

[25] G.S. Rushbrooke, G. Stell and J.S. Hole, Mol. Phys. 26, 1199 (1973).

[26] J. Winkelmann, Fluid Phase Equilibria 11, 207 (1983).

[27] B. Madan and T. Keyes, J. Chem. Phys. 98, 3342 (1993).

[28] H.J. Raverche, J. Chem. Phys. 55, 2242 (1971); D.C. Wallace, J. Chem. Phys. 87, 2282 (1987);

A. Baranyai and D.J. Evans, Phys. Rev. A 42, 849 (1990); I. Borzsak and A. Baranyai, Chem. Phys. 165, 227 (1992).

[29] B.B. Laird and A.D.J. Haymet, Phys. Rev. A 45, 5680 (1992).

[30] S.T. Lin, M.(Blanco, W.A. Goddard, J. Chem. Phys. 119, 11792 (2003).

[31] W.L. Jorgensen, J. Phys. Chem. 90, 1276 (1986).

[32] F. Hirata and R.M. Levy, J. Phys. Chem. 91, 4788 (1987).

[33] E. Guàrdia, G. Sesé, and J.A. Padró, J. Mol. Phys. 62, 1 (1994).

[34] R. Palomar and G. Sesé, J. Phys. Chem. B, 109, 499 (2005).

[35] R. Palomar and G. Sesé, J. Chem. Phys. 133, 044501 (2010).

[36] D. Fragidiakis and C.M. Roland, J. Chem. Phys. 138, 12A502 (2013).

[37] H.J.C. Berendsen, J.P.M. Postma, W.F. van Gunsteren, A. DiNola, and J.R. Haak, J. Chem. Phys. 81, 3684 (1984).

[38] T.C. Beutler, A.E. Mark, R.C. Chaik, P.R. Gerber, and W.F. van Gunsteren, Chem. Phys. Letters 222, 529 (1994). 
M.R. Shirts, J.W. Pitera, W.C. Swope, and V.S. Pande, J. Chem. Phys. 119, 5740 (2003); M.R. Shirts and V.S. Pande, J. Chem. Phys. 122, 134508 (2005); T. Steinbrecher, D.L. Mobley and D.A. Case, J. Chem. Phys. 127, 214108 (2007).

[40] Wu-X. Li, T. Keyes, R.L.Murry and J.T.Fourkas, J. Chem. Phys. 109, 9096 (1998); R.L.Murry, J.T.Fourkas, Wu-X. Li and T. Keyes, J. Chem. Phys. 110, 10410 (1999); R.L.Murry, J.T.Fourkas, Wu-X. Li and T. Keyes, J. Chem. Phys. 110, 10423 (1999).

[41] R. Palomar, PhD Thesis, Technical University of Catalonia (UPC) http://www.tdx.cat/TDX0630108-120910 (2007).

[42] G. Sesé, J. Ortiz de Urbina and R. Palomar, J. Chem. Phys. 137, 114502 (2012).

[43] J.R. Errington, T.M. Truskett and J. Mittal, J. Chem. Phys. 125, 244502 (2006).

[44] Z. Yan, S.V. Buldyrev and H.E. Stanley, Phys. Rev. E 78, 051201 (2008).

[45] K. Seki and B. Bagchi, J. Chem. Phys. 143, 194110(2015).

[46] P. Gallo and M. Rovere, Phys. Rev. E 91, 012107 (2015).

[47] R. Palomar and G. Sesé, Phys. Rev. E 75, 011505 (2007).

[48] R. Palomar and G. Sesé, J. Chem. Phys. 129, 064505 (2008).

[49] Y.D. Fomin, V.N. Ryzhov and N.V. Gribova, Phys Rev E 81, 061201 (2010).

[50] A. Banerjee, M.K. Nandi, and S. M. Bhattacharyya, J. Chem. Sci. 129, 793 (2017).

[51] J. P. Boon and S.Yip, Molecular Hydrodynamics (Mc Graw-Hill, New York, 1980).

[52] L. Sjögren and A. Sjölander, J. Phys. C: Solid State Phys. 12, 4369 (1979).

[53] U. Balucani and M. Zoppi, Dynamics of the Liquid State (Clarendon Press, Oxford, 1994).

[54] E. Ruckenstein and H. Liu, Ind. Eng. Chem. Res. 36, 3927 (1997).

[55] T. Keyes, Phys. Rev.E 62, 7905 (2000).

[56] P. Moore and T. Keyes, J. Chem. Phys. 100, 6709 (1994).

[57] S.D. Bembenek and B.B. Laird, Phys. Rev. Lett. 74, 936 (1995).

[58] E. La Naye, A. Scala, F. Starr, F. Sciortino and H.E. Stanley, Phys. Rev. Lett. 84, 174504 (2000).

[59] S. Jiu-Xun, C. Ling-Cang, W. Qiang, and J. Fu-Qian, Commun. Theor. Phys. 41, 400 (2004).

[60] A.K. Singh, T.K. Dey, and S.K. Sinha, Pramana - J. Phys. 34, No. 2, 123 (1990).

[61] A. Tani, D. Henderson, J.A. Barker, and C.E. Hecht, Mol. Phys. 48, 863 (1983). 


\begin{tabular}{cccc}
\hline \hline Site & $\mathrm{q}(\mathrm{e})$ & $\sigma(\AA)$ & $\varepsilon /\left(k_{B}\right)$ \\
\hline $\mathrm{O}$ & -0.323 & 3.070 & 85.55 \\
$\mathrm{Me}$ & 0.323 & 3.775 & 104.17 \\
\hline \hline
\end{tabular}

Table I. Potential parameters for the $\mathrm{MeO}$ molecule.

\begin{tabular}{|c|c|c|c|c|c|c|}
\hline Model & $A_{H}$ & $A_{R}$ & $A_{1 S W}$ & $A_{2 S W}$ & $A_{S W} A_{D D}$ & $S$ \\
\hline HS & -3.86 & -3.12 & -3.53 & $-4.33 \cdot 10^{-2}$ & & -10.4626 .88 \\
\hline $\mathrm{HE}$ & -3.80 & -3.12 & -3.67 & $-4.20 \cdot 10^{-2}$ & $-2.53-0.95$ & -10.3426 .48 \\
\hline
\end{tabular}

Table II. Free energy (in kcal/mol) and entropy (in cal mol ${ }^{-1} \mathrm{~K}^{-1}$ ) obtained by using a perturbation theory approach. Molecules are modeled as hard spheres (HS) or hard ellipsoids (HE) at T=298 K. Partial terms in eq. (11) are also listed. 


\begin{tabular}{ccccc}
\hline \hline $\mathrm{T}(\mathrm{K})$ & $S$ & $S^{T}$ & $S^{R}$ & $q$ \\
\hline 298 & 29.19 & 18.86 & 10.33 & 0.462 \\
268 & 27.82 & 17.98 & 9.84 & 0.429 \\
238 & 26.25 & 16.93 & 9.32 & 0.394 \\
218 & 25.06 & 16.17 & 8.89 & 0.385 \\
208 & 24.42 & 15.75 & 8.67 & 0.351 \\
198 & 23.74 & 15.29 & 8.45 & 0.334 \\
178 & 22.25 & 14.32 & 7.93 & 0.305 \\
158 & 20.53 & 13.18 & 7.35 & 0.268 \\
138 & 18.54 & 11.86 & 6.68 & 0.227 \\
123 & 16.80 & 10.69 & 6.11 & 0.191 \\
\hline \hline
\end{tabular}

Table III. Total entropy $(S)$, translational $\left(S^{T}\right)$ and rotational $\left(S^{R}\right)$ contributions in cal mol ${ }^{-1}$ $\mathrm{K}^{-1}$, and values for the gas-like $q$ parameter at different temperatures.

Figure 1. Integrand in eq. (4) versus $\alpha$.

Figure 2. COM Radial distribution function of liquid $\mathrm{MeO}$ and that of the hard sphere fluid at the same density and temperature. Hard spheres diameter is $d=3.85 \AA$. 


\begin{tabular}{cccc}
\hline \hline $\mathrm{T}(\mathrm{K})$ & $D$ & $D_{B}$ & $D_{C}$ \\
\hline 298 & $5.69 \cdot 10^{-5}$ & $7.67 \cdot 10^{-5}$ & $2.20 \cdot 10^{-4}$ \\
268 & $4.32 \cdot 10^{-5}$ & $6.54 \cdot 10^{-5}$ & $1.27 \cdot 10^{-4}$ \\
238 & $2.94 \cdot 10^{-5}$ & $5.32 \cdot 10^{-5}$ & $6.57 \cdot 10^{-5}$ \\
218 & $2.17 \cdot 10^{-5}$ & $4.74 \cdot 10^{-5}$ & $4.00 \cdot 10^{-5}$ \\
208 & $1.82 \cdot 10^{-5}$ & $4.34 \cdot 10^{-5}$ & $3.13 \cdot 10^{-5}$ \\
198 & $1.45 \cdot 10^{-5}$ & $3.94 \cdot 10^{-5}$ & $2.29 \cdot 10^{-5}$ \\
178 & $9.42 \cdot 10^{-6} 3.36 \cdot 10^{-5}$ & $1.31 \cdot 10^{-5}$ \\
158 & $5.31 \cdot 10^{-6}$ & $2.86 \cdot 10^{-5}$ & $6.52 \cdot 10^{-6}$ \\
138 & $1.98 \cdot 10^{-6}$ & $2.34 \cdot 10^{-5}$ & $2.16 \cdot 10^{-6}$ \\
123 & $8.30 \cdot 10^{-7}$ & $1.99 \cdot 10^{-5}$ & $8.66 \cdot 10^{-7}$ \\
\hline \hline
\end{tabular}

Table IV. Center-of-mass diffusion coefficients $(D)$ and binary contributions $\left(D_{B}\right)$ from ([35]). Mode-coupling contributions $\left(D_{C}\right)$ as defined in eq. (51) at several temperatures. Diffusion coefficients are given in $\mathrm{cm}^{2} / \mathrm{s}$.

Figure 3. Densities of states obtained by INM anlysis. Total (continuous line), translational (dotted-dashed line) and rotational (dotted line) DOS for (a) $T=298 \mathrm{~K}$ and (b) $T=123 \mathrm{~K}$. Imaginary frequencies are shown in the negative $\omega$ axis.

Figure 4. Reduced pair correlation entropy $s_{2}^{*}$ versus reduced excess entropy $s_{\text {exc }}^{*}$. The fitting line is $s_{2}^{*}=0.84 s_{e x c}^{*}$

Figure 5. Reduced diffusion coefficient $\left(D^{*}\right)$, in logarithmic scale, versus reduced excess entropy $s_{\text {exc. }}^{*}$. The slope of the fitting line is $b=1.08$.

Figure 6. Reduced excess entropy (dots) and reduced binary entropy (squares). 
Figure 7. Reduced binary diffusivity (eq. (52)) against reduced excess entropy $s_{B}^{*}$ (eq. (53)). Continuous line is a linear fit with a slope $b_{1}=0.75$.

Figure 8. Reduced coupling diffusivity $D_{C}^{*}$ against $s_{e x c}^{*} / s_{B}^{*}$. Continuous line is the result of a fit to eq. (55) with $b_{2}=-12.3$.

Figure 9. Reduced excess entropy against the fraction of unstable modes $\left(f_{u}\right)$ in the translational DOS of COM. Inset: Reduced excess entropy versus $\ln \left(f_{u}\right)$. 


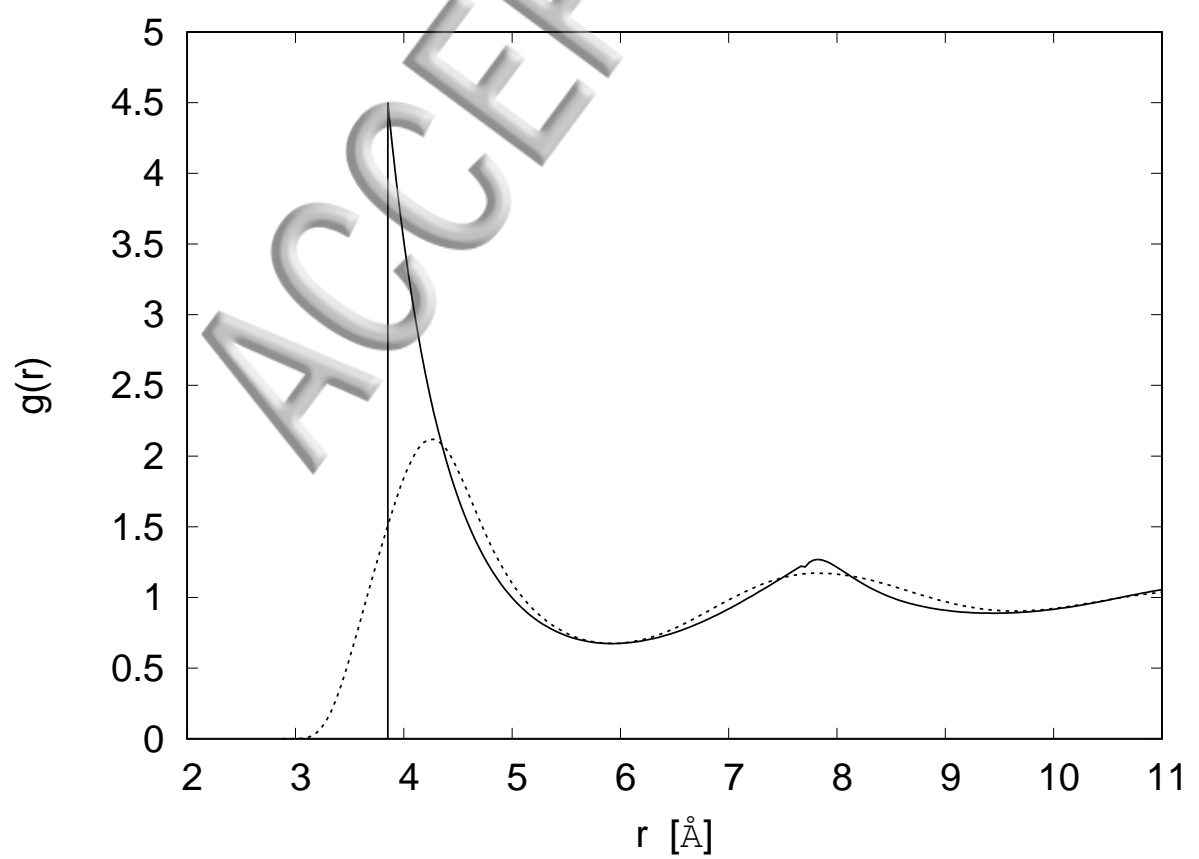




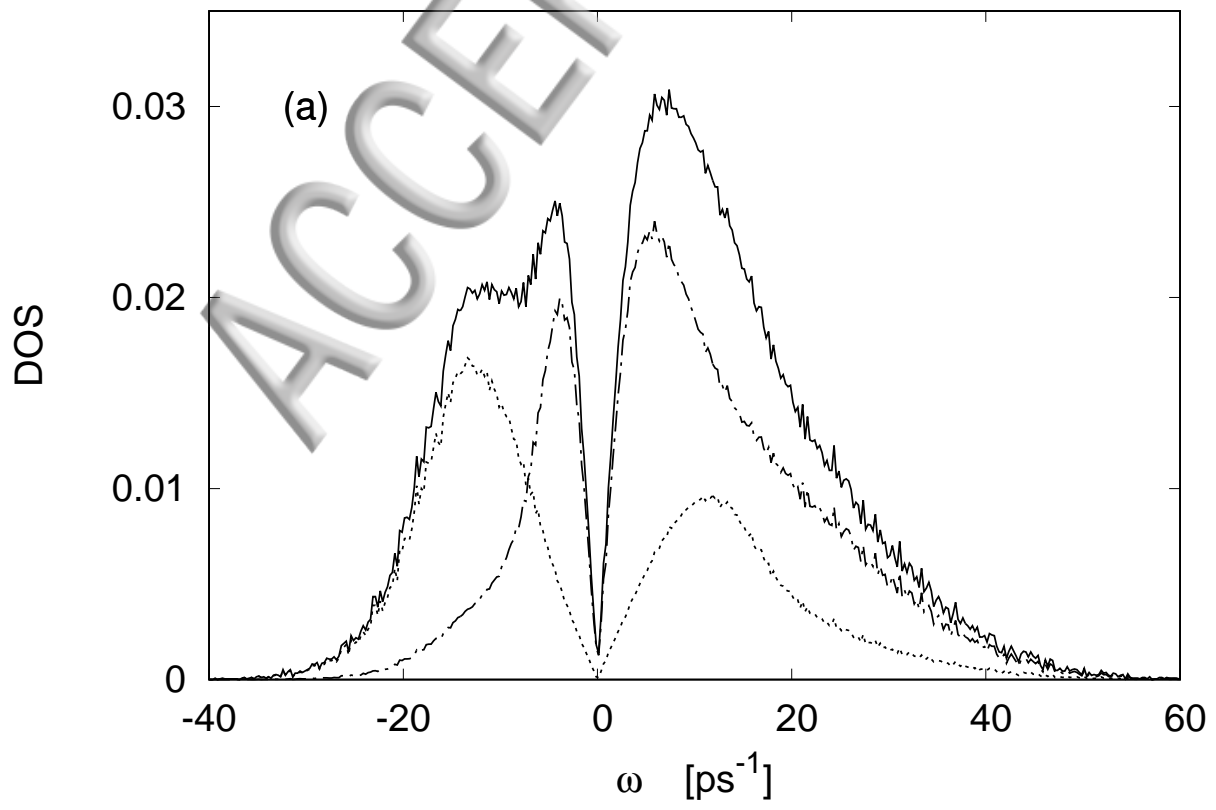




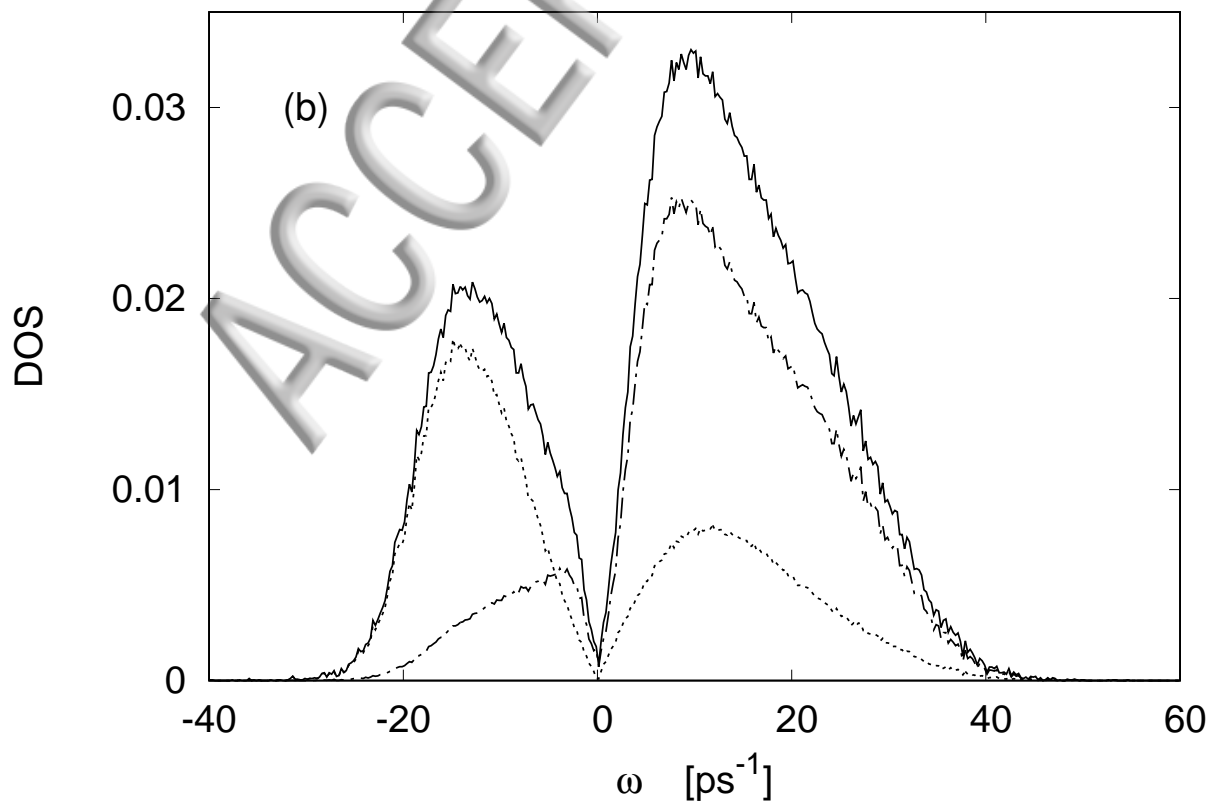




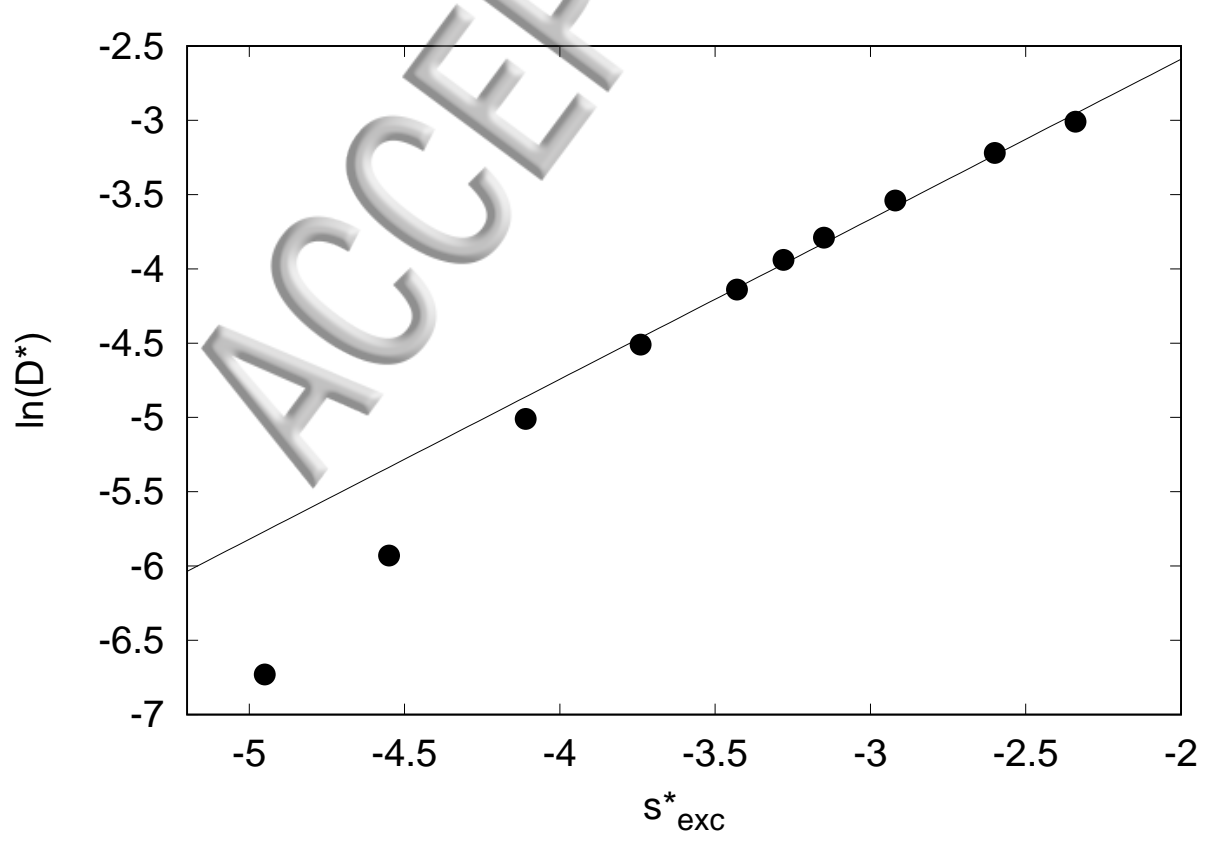




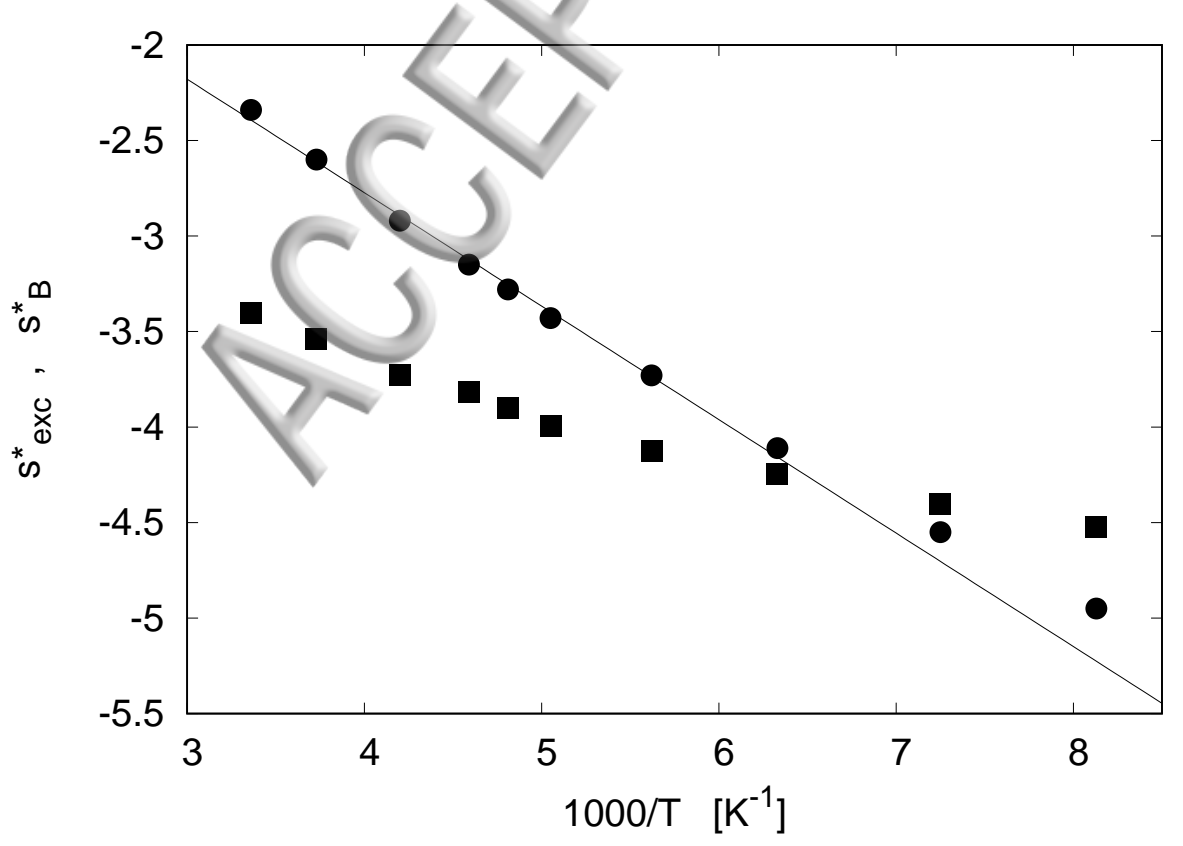




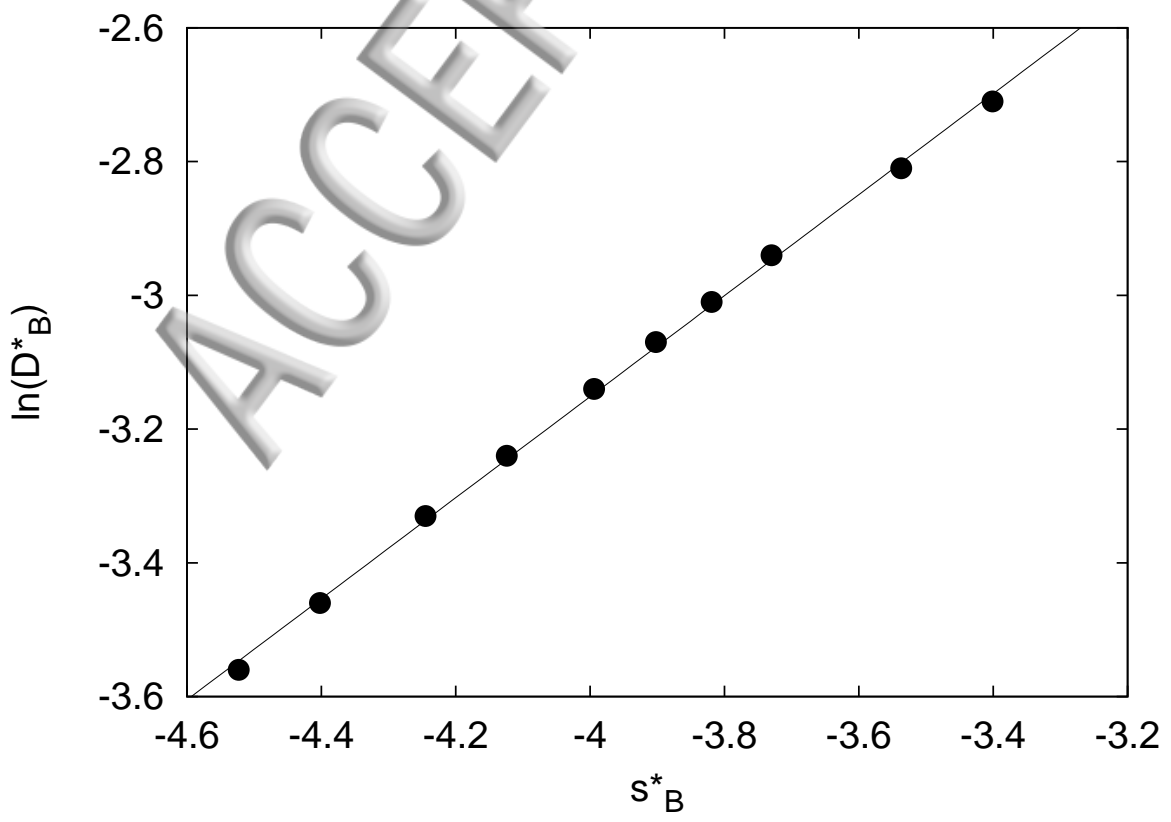




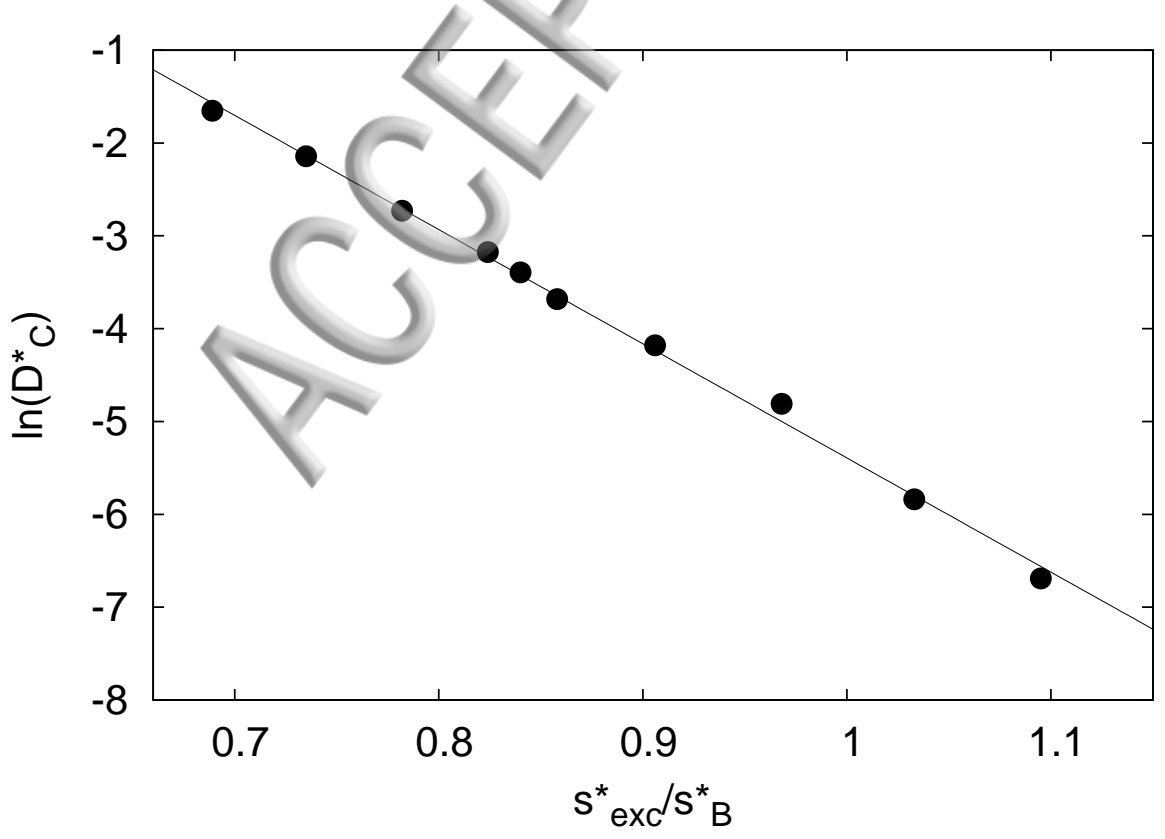




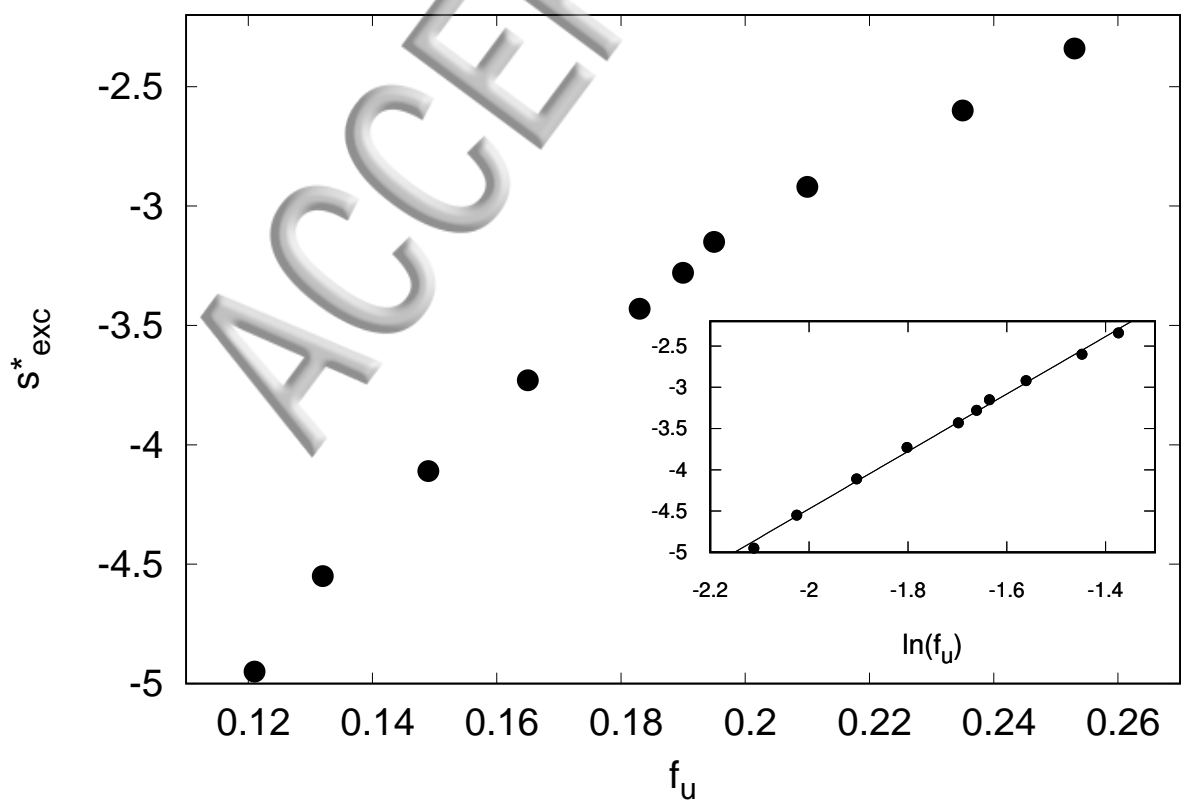

